\title{
On the regularity of solutions of the boundary value problem without initial condition for Schrödinger systems in domain with conical points
}

\author{
Nguyen Thi Lien ${ }^{1 *}$ and Nguyen Manh Hung ${ }^{2}$
}

\section{"Correspondence:}

Lienhnue@gmail.com

'Department of Mathematics,

Hanoi National University of

Education, Hanoi, Vietnam

Full list of author information is

available at the end of the article

\begin{abstract}
In this paper, we deal with the boundary value problems without initial condition for Schrödinger systems in cylinders. We establish several results on the regularity of the solutions.

MSC: $35 \mathrm{Q} 41 ; 35 \mathrm{~B} 65 ; 35 \mathrm{D} 30$

Keywords: regularity of generalized solution; problems without initial condition; cylinders
\end{abstract}

\section{Introduction}

The theory of initial boundary value problems for partial differential equations and systems in nonsmooth domains has attracted the attention of many researchers. These problems for Schrödinger systems in domain with conical points are considered in [1,2], in which authors consider the existence, the uniqueness and the regularity of solutions of the mentioned problems. While problems without initial conditions arise when describing different nonstationary processes in nature under the hypothesis that the initial condition practically has no influence at the present time. These problems are investigated in many works, see [3-6] for example, with results only about the well-posedness. In [7], we studied the boundary value problem without initial condition for Schrödinger systems in domain with conical points. By studying the corresponding problem with initial condition $t=h$, then passing to the limits $h \rightarrow-\infty$, we obtained the existence and uniqueness of the solution. In the present paper, we continue considering the mentioned problem and our main aim here is to study the regularity of the solution.

There are four sections in this paper. In Section 1, we set the problem and recall an obvious result about the unique existence of solution. Afterwards, in Sections 2 and 3, by a similar method to $[1,2]$, we give results on the regularity of problems with initial condition $t=h$ for Schrödinger systems in domains with conical points. Then, by letting $h \rightarrow-\infty$, the smoothness of the generalized solution of our problem is obtained in Section 4 .

\section{Setting problem and obvious result}

Let $\Omega$ be a bounded domain in $\mathbb{R}^{n}(n \geq 2)$ with boundary $S=\partial \Omega$. Assume that $S$ is an infinitely differentiable surface everywhere, except for the coordinate origin, in a neigh- 
borhood $U_{0}$ satisfying $\Omega \cap U_{0}$ and coincides with the cone $K=\{x: x /|x| \in G\}$, where $G$ is a smooth domain on the unit sphere $S^{n-1}$. For $a<b$, set $\Omega_{a}^{b}=\Omega \times(a, b), S_{a}^{b}=S \times(a, b)$. If $(a, b)=\mathbb{R}$, we use $\Omega_{\mathbb{R}}$ to refer to $\Omega_{-\infty}^{\infty}$ and $S_{\mathbb{R}}$ to refer to $S_{-\infty}^{\infty}$. For each multi-index $\alpha=\left(\alpha_{1}, \ldots, \alpha_{n}\right) \in \mathbb{N}^{n}$, set $|\alpha|=\alpha_{1}+\cdots+\alpha_{n}$ and $\partial^{\alpha}=\partial_{x_{1}}^{\alpha_{1}} \cdots \partial_{x_{n}}^{\alpha_{n}}$.

Denote $u(x, t)=\left(u_{1}(x, t), \ldots, u_{s}(x, t)\right), D^{\alpha} u=\left(D^{\alpha} u_{1}, \ldots, D^{\alpha} u_{s}\right),\left|D^{\alpha} u\right|^{2}=\sum_{i=1}^{s}\left|D^{\alpha} u_{i}\right|^{2}$ and $u_{t^{j}}=\left(\frac{\partial^{j} u_{1}}{\partial \dot{t}^{j}}, \ldots, \frac{\partial^{j} u_{s}}{\partial \dot{t}^{\prime}}\right),\left|u_{t}\right|^{2}=\sum_{i=1}^{s}\left|\frac{\partial^{j} u_{i}}{\partial t^{j}}\right|^{2}$.

Let us introduce some functional spaces (see [7]) used in this paper.

We use $H^{k}(\Omega)$ to denote the space of $s$-dimensional vector functions defined in $\Omega$ with the norm

$$
\|u\|_{H^{k}(\Omega)}=\left(\sum_{|\alpha|=0}^{k} \int_{\Omega}\left|D^{\alpha} u\right|^{2} d x\right)^{\frac{1}{2}} .
$$

Denote by $H^{k, l}\left(\Omega_{a}^{b}\right)$ the space consisting of all vector functions $u: \Omega_{a}^{b} \longrightarrow \mathbb{C}^{s}$ satisfying

$$
\|u\|_{H^{k, l}\left(\Omega_{a}^{b}\right)}=\left(\int_{\Omega_{a}^{b}}\left(\sum_{|\alpha|=0}^{k}\left|D^{\alpha} u\right|^{2}+\sum_{j=1}^{l}\left|u_{t^{j}}\right|^{2}\right) d x d t\right)^{\frac{1}{2}},
$$

and $H^{k, l}\left(-\gamma, \Omega_{a}^{b}\right)$ is the space of vector functions with norm

$$
\|u\|_{H^{k, l}\left(-\gamma, \Omega_{a}^{b}\right)}=\left(\int_{\Omega_{a}^{b}}\left(\sum_{|\alpha|=0}^{k}\left|D^{\alpha} u\right|^{2}+\sum_{j=1}^{l}\left|u_{t}\right|^{2}\right) e^{-2 \gamma t} d x d t\right)^{\frac{1}{2}} .
$$

In particular,

$$
\|u\|_{H^{k, 0}\left(-\gamma, \Omega_{a}^{b}\right)}=\left(\sum_{|\alpha|=0}^{k} \int_{\Omega_{a}^{b}}\left|D^{\alpha} u\right|^{2} e^{-2 \gamma t} d x d t\right)^{\frac{1}{2}} .
$$

Especially, we set $L_{2}\left(-\gamma, \Omega_{a}^{b}\right)=H^{0,0}\left(-\gamma, \Omega_{a}^{b}\right)$.

$H_{\beta}^{l, k}\left(-\gamma, \Omega_{a}^{b}\right)$ is the space of all functions $u(x, t)$ which have generalized derivatives $D^{\alpha} u$, $\frac{\partial^{j} u}{\partial \dot{t}^{\prime}},|\alpha| \leq l, 1 \leq j \leq k$, satisfying

$$
\|u\|_{H_{\beta}^{l, k}\left(-\gamma, \Omega_{a}^{b}\right)}^{2}=\int_{\Omega_{a}^{b}}\left(\sum_{\alpha=0}^{l} r^{2(\beta+|\alpha|-l)}\left|D^{\alpha} u\right|^{2}+\sum_{j=1}^{k}\left|u_{t}\right|^{2}\right) e^{-2 \gamma t} d x d t<\infty
$$

$H_{\beta}^{l}\left(-\gamma, \Omega_{a}^{b}\right)$ is the space of all functions $u(x, t)$ which have generalized derivatives $D^{\alpha} u_{t}$, $|\alpha| \leq l, 1 \leq j \leq l$, satisfying

$$
\|u\|_{H_{\beta}^{l}\left(-\gamma, \Omega_{a}^{b}\right)}^{2}=\int_{\Omega_{a}^{b}} \sum_{\alpha+j=0}^{l} r^{2(\beta+|\alpha|+j-l)}\left|D^{\alpha} u_{t}\right|^{2} e^{-2 \gamma t} d x d t<\infty .
$$

Denote by $\stackrel{\circ}{H}^{k, l}\left(-\gamma, \Omega_{a}^{b}\right)$ the completion of infinitely differentiable vector functions vanishing near $S_{a}^{b}$ with respect to $H^{l, k}\left(-\gamma, \Omega_{a}^{b}\right)$ norm. 
Now we introduce a differential operator of order $2 m$

$$
L(x, t, D)=\sum_{|p|,|q|=0}^{m}(-1)^{|p|} D^{p}\left(a_{p q}(x, t) D^{q}\right),
$$

where $a_{p q}$ are $s \times s$ matrices with the bounded complex-valued components in $\bar{\Omega}_{\mathbb{R}}, a_{p q}=$ $a_{q p}^{*}\left(a_{q p}^{*}\right.$ is the transposed conjugate matrix to $\left.a_{p q}\right)$. Set

$$
B(t, u, v)=\sum_{|p|,|q|=0}^{m} \int_{\Omega} a_{p q} D^{q} u \overline{D^{p} v} d x, \quad t \in \mathbb{R} .
$$

We assume further that the form $B(t, \cdot, \cdot)$ satisfies the following condition: there exists a constant $\mu_{0}>0$ independent of $t$ and $u$ such that

$$
B(t, u, u) \geq \mu_{0}\|u(\cdot, t)\|_{H^{m}(\Omega)}^{2}
$$

for all $u \in \stackrel{\circ}{H}^{m}(\Omega)$ and a.e. $t \in \mathbb{R}$, where $\stackrel{\circ}{H}^{m}(\Omega)$ is the closure in $H^{m}(\Omega)$ of infinitely differentiable complex $s$-dimensional vector functions with compact support in $\Omega$.

Now we consider the following problem in the cylinder $\Omega_{\mathbb{R}}$ :

$$
\begin{aligned}
& (-1)^{m-1} i L(x, t, D) u-u_{t}=f(x, t) \quad \text { in } \Omega_{\mathbb{R}}, \\
& \left.\frac{\partial^{j} u}{\partial \nu^{j}}\right|_{S_{\mathbb{R}}}=0, \quad j=0, \ldots, m-1,
\end{aligned}
$$

where $v$ is the unit vector of outer normal to the surrounding surface $S_{\mathbb{R}}$.

Let $f \in L_{2}\left(-\gamma, \Omega_{\mathbb{R}}\right)$, a complex vector-valued function $u \in \stackrel{\circ}{H}^{m, 0}\left(-\gamma, \Omega_{\mathbb{R}}\right)$ is called a generalized solution of problem (2.2)-(2.3) if and only if, for any $T>0$, the equality

$$
(-1)^{m-1} i \int_{-\infty}^{T} B(t, u, \eta) d t+\int_{\Omega_{-\infty}^{T}} u \overline{\eta_{t}} d x d t=\int_{\Omega_{-\infty}^{T}} f \bar{\eta} d x d t
$$

holds for all $\eta \in \stackrel{\circ}{H}^{m, 1}\left(\gamma, \Omega_{\mathbb{R}}\right), \eta(x, t)=0$ with $t \geq T$.

For any $h \in \mathbb{R}$, we also consider the following problem with initial condition corresponding to problem (2.2)-(2.3):

$$
\begin{aligned}
& (-1)^{m-1} i L(x, t, D) v-v_{t}=f(x, t) \quad \text { in } \Omega_{h}^{\infty}, \\
& \left.v\right|_{t=h}=0, \quad x \in \Omega, \\
& \left.\frac{\partial^{j} v}{\partial \nu^{j}}\right|_{S_{h}^{\infty}}=0, \quad j=0, \ldots, m-1 .
\end{aligned}
$$

A function $v \in \stackrel{\circ}{H}^{m, 0}\left(-\gamma, \Omega_{h}^{\infty}\right)$ is a generalized solution of problem (2.5)-(2.7) if and only if, for any $T>0$, we have

$$
(-1)^{m-1} i \int_{h}^{T} B(t, v, \eta) d t+\int_{\Omega_{h}^{T}} v \overline{\eta_{t}} d x d t=\int_{\Omega_{h}^{T}} f \bar{\eta} d x d t
$$

for all $\eta \in \stackrel{\circ}{H}^{m, 1}\left(\gamma, \Omega_{h}^{\infty}\right), \eta(x, t)=0$ for all $t \geq T$. 
We recall the results about the solvability of problem (2.2)-(2.3) proved in [7].

Theorem 2.1 Assume that:

(i) $\sup \left\{\left|\frac{\partial a_{p q}}{\partial t}\right|:(x, t) \in \Omega_{\mathbb{R}}, 0 \leq|p|,|q| \leq m\right\}=\mu<\infty$;

(ii) $\left|\frac{\partial a_{p q}}{\partial t}\right| \leq \mu_{1} \cdot e^{2 \gamma t}$, for all $(x, t) \in \Omega_{\mathbb{R}}, 0 \leq|p|,|q| \leq m$;

(iii) $f, f_{t} \in L_{2}\left(-\gamma, \Omega_{\mathbb{R}}\right)$,

where $\mu, \mu_{1}$ are positive constants. Then for all $\gamma>\gamma_{0}=\frac{m^{\star} \mu}{2 \mu_{0}}, m^{\star}=\sum_{|\alpha| \leq m} 1$, there exists a uniquely generalized solution $u \in \stackrel{\circ}{H}^{m, 0}\left(-\gamma, \Omega_{\mathbb{R}}\right)$ of problem (2.2)-(2.3) satisfying

$$
\|u\|_{H^{m, 0}\left(-\gamma, \Omega_{\mathbb{R}}\right)}^{2} \leq C\left[\|f\|_{L_{2}\left(-\gamma, \Omega_{\mathbb{R}}\right)}^{2}+\left\|f_{t}\right\|_{L_{2}\left(-\gamma, \Omega_{\mathbb{R}}\right)}^{2}\right]
$$

\section{The regularity with respect to time variable of the problem with initial condition}

The regularity of problem (2.2)-(2.3) is implied by analogous property for problem (2.5)(2.7). In [1], the regularity with respect to time variable of problem (2.5)-(2.7) is studied in the case $h=0$ and $f_{t^{k}} \in L^{\infty}\left(0, \infty ; L_{2}(\Omega)\right)$. Now, by a similar method, we consider the problem in the case $h \in \mathbb{R}$ and $f_{t^{k}} \in L_{2}\left(-\gamma, \Omega_{h}^{\infty}\right)$. Moreover, we also show that the constants $C$ in all prior estimates do not depend on $h$.

Theorem 3.1 Assume that $l \in \mathbb{N}$ and there exists $\mu>0$ such that

(i) $\sup \left\{\left|\frac{\partial a_{p q}}{\partial t}\right|:(x, t) \in \Omega_{\mathbb{R}}, 0 \leq|p|,|q| \leq m,\right\}=\mu<\infty,\left|\frac{\partial^{k} a_{p q}}{\partial t^{k}}\right| \leq \mu_{2}, \mu_{2}>0$, for $2 \leq k \leq l+1$

(ii) $f_{t^{k}} \in L_{2}\left(-\gamma, \Omega_{h}^{\infty}\right)$, for $k \leq l+1, f_{t^{k}}(x, h)=0$ for all $x \in \Omega, 0 \leq k \leq l$.

Then for all $\gamma>(2 l+1) \gamma_{0}$, the solution $v \in \stackrel{\circ}{H}^{m, 0}\left(-\gamma, \Omega_{\mathbb{R}}\right)$ of problem (2.5)-(2.7) has derivatives with respect to $t$ up to order $l$ satisfying $v_{t^{k}} \in H^{m, 0}\left(-\gamma, \Omega_{h}^{\infty}\right), \forall k=\overline{0, l}$, such that

$$
\left\|v_{t^{k}}\right\|_{H^{m, 0}\left(-\gamma, \Omega_{h}^{\infty}\right)}^{2} \leq C \sum_{m_{1}=0}^{k+1}\left\|f_{t^{m_{1}}}\right\|_{L_{2}\left(-\gamma, \Omega_{h}^{\infty}\right)}^{2},
$$

where the constant $C$ does not depend on $v, f, h$.

Proof Suppose that $\left\{\varphi_{k}\right\}_{k=1}^{\infty}$ is an orthogonal basis of $H^{m}(\Omega)$ which is orthonormal in $L_{2}(\Omega)$. For any $N \in \mathbb{N}$, the approximating solutions can be found in the form $v^{N}(x, t)=$ $\sum_{k=0}^{N} C_{k}^{N}(t) \varphi_{k}(x)$ where $\left(C_{k}^{N}(t)\right)_{k=1}^{N}$ is the solution of the ordinary differential system

$$
\begin{aligned}
& (-1)^{m-1} i B\left(t, v^{N}, \varphi_{k}\right)-\int_{\Omega} v_{t}^{N} \overline{\varphi_{k}} d x=\int_{\Omega} f \overline{\varphi_{k}} d x, \\
& C_{k}^{N}(h)=0, \quad k=1, \ldots, N .
\end{aligned}
$$

From the assumptions it follows that the coefficients $C_{k}^{N}(t)$, defined uniquely by (3.2), have derivatives up to order $1+1$ and $v^{N}(x, h)=0$. It is very easy to check that $D^{p} v^{N}(x, h)=0$, $\forall 0 \leq|p| \leq m, x \in \Omega$.

Step 1: Prove that $D^{p} v_{t^{k}}^{N}(x, h)=0$ for all $0 \leq k \leq l, 0 \leq|p| \leq m, x \in \Omega$. 
Taking the derivative $(k-1)$ times of both sides of (3.2) with respect to $t$, then multiplying both sides by $\frac{d^{k}}{d t^{k}} \overline{\left(C_{k}^{N}(t)\right)}$ and taking the sum with respect to $k$ from 1 to $N$, we arrive at

$$
\begin{aligned}
& -i \int_{\Omega}\left|v_{t^{k}}^{N}\right|^{2} d x+(-1)^{m} \sum_{|p|,|q|=0}^{m} \int_{\Omega} a_{p q} D^{q} v_{t^{k-1}}^{N} \overline{D^{p} v_{t^{k}}^{N}} d x \\
& \quad=i \int_{\Omega} f_{t^{k-1}} \overline{v_{t^{k}}^{N}} d x+(-1)^{m-1} \sum_{|p|,|q|=0}^{m} \sum_{s=0}^{k-2}\left(\begin{array}{c}
k-1 \\
s
\end{array}\right) \int_{\Omega} \frac{\partial^{k-s-1} a_{p q}}{\partial t^{k-s-1}} D^{q} v_{t^{s}}^{N} \overline{D^{p} v_{t^{k-s-1}}^{N}} d x .
\end{aligned}
$$

Because of the fact that $f_{t^{k}}(x, h)=0$ for all $x \in \Omega, 0 \leq k \leq l-1$, using induction with respect to $k$ from (3.4) we obtain $D^{p} v_{t^{k}}^{N}(x, h)=0$ for all $0 \leq k \leq l, 0 \leq|p| \leq m, x \in \Omega$.

Step 2: A priori estimate for $v_{t^{k}}^{N}$.

For $k=l+1$, adding (3.4) to its complex conjugate, integrating with respect to $t$ from $h$ to $\tau$, and then integrating by parts, we get

$$
\begin{array}{rl}
(-1)^{m} & B\left(\tau, v_{t^{l}}^{N}(x, \tau), v_{t^{l}}^{N}(x, \tau)\right) \\
= & (-1)^{m} \sum_{|p|,|q|=0}^{m} \int_{\Omega_{h}^{\tau}} \frac{\partial a_{p q}}{\partial t} D^{q} v_{t^{l}}^{N} \overline{D^{p} v_{t^{l}}^{N}} d x d t \\
& +(-1)^{m} 2 \operatorname{Re} \sum_{|p|,|q|=0}^{m} l \cdot \int_{\Omega_{h}^{\tau}} \frac{\partial a_{p q}}{\partial t} D^{q} v_{t^{l}}^{N} \overline{D^{p} v_{t^{l}}^{N}} d x d t \\
& +(-1)^{m} 2 \operatorname{Re} \sum_{|p|,|q|=0}^{m} \sum_{s=0}^{l-1}\left(\begin{array}{l}
l \\
s
\end{array}\right) \int_{\Omega_{h}^{\tau}} \frac{\partial^{l-s+1} a_{p q}}{\partial t^{l-s+1}} D^{q} v_{t^{s}}^{N} \overline{D^{p} v_{t^{l}}^{N}} d x d t \\
& +(-1)^{m} 2 \operatorname{Re} \sum_{|p|,|q|=0}^{m} \sum_{s=0}^{l-2}\left(\begin{array}{l}
l \\
s
\end{array}\right) \int_{\Omega_{h}^{\tau}} \frac{\partial^{l-s} a_{p q}}{\partial t^{l-s}} D^{q} v_{t^{s+1}}^{N} \overline{D^{p} v_{t^{l}}^{N}} d x d t \\
& -(-1)^{m} 2 \operatorname{Re} \sum_{|p|,|q|=0}^{m} \sum_{s=0}^{l-1}\left(\begin{array}{l}
l \\
s
\end{array}\right) \int_{\Omega} \frac{\partial^{l-s} a_{p q}}{\partial t^{l-s}}(x, \tau) D^{q} v_{t^{s}}^{N}(x, \tau) \overline{D^{p} v_{t^{l}}^{N}(x, \tau)} d x \\
& +2 \operatorname{Im} \int_{\Omega} f_{t^{l}}(x, \tau) \overline{v_{t^{l}}^{N}(x, \tau)} d x-2 \operatorname{Im} \int_{\Omega_{h}^{\tau}} f_{t^{l+1}} \overline{v_{t^{l}}^{N}} d x d t .
\end{array}
$$

We denote by $I, I I, I I I, I V, V$, and $V I$ the terms from the first, second, third, fourth, fifth, sixth, and seventh, respectively, of the right-hand sides of (3.5). We will give estimations for these terms. Using Cauchy's inequality, for any $\epsilon_{1}>0$, we have

$$
\begin{aligned}
& I \leq \frac{\mu}{2} \sum_{|p|,|q|=0}^{m} \int_{\Omega_{h}^{\tau}}\left(\left|D^{q} v_{t^{l}}^{N}\right|^{2}+\left|D^{p} v_{t^{l}}^{N}\right|^{2}\right) d x d t=\mu m^{\star} \int_{h}^{\tau}\left\|v_{t^{l}}^{N}(\cdot, t)\right\|_{H^{m}(\Omega)}^{2} d t, \\
& I I \leq 2 l \mu m^{\star} \int_{h}^{\tau}\left\|v_{t^{l}}^{N}(\cdot, t)\right\|_{H^{m}(\Omega)}^{2} d t \\
& I I I \leq \mu_{2} m^{\star}\left(2^{l}-1\right) \epsilon_{1} \int_{h}^{\tau}\left\|v_{t^{l}}^{N}(\cdot, t)\right\|_{H^{m}(\Omega)}^{2} d t+C_{1} \sum_{s=0}^{l-1} \int_{h}^{\tau}\left\|v_{t^{s}}^{N}(\cdot, t)\right\|_{H^{m}(\Omega)}^{2} d t, \\
& I V \leq \mu_{2} m^{\star}\left(2^{l}-l-1\right) \epsilon_{1} \int_{h}^{\tau}\left\|v_{t^{l}}^{N}(\cdot, t)\right\|_{H^{m}(\Omega)}^{2} d t+C_{1} \sum_{s=0}^{l-1} \int_{h}^{\tau}\left\|v_{t^{s}}^{N}(\cdot, t)\right\|_{H^{m}(\Omega)}^{2} d t,
\end{aligned}
$$




$$
\begin{gathered}
V \leq \mu_{2} m^{\star}\left(2^{l}-1\right) \epsilon_{1}\left\|v_{t^{s}}^{N}(\cdot, \tau)\right\|_{H^{m}(\Omega)}^{2}+C_{1} \sum_{s=0}^{l-1}\left\|v_{t^{s}}^{N}(\cdot, \tau)\right\|_{H^{m}(\Omega)}^{2}, \\
V I \leq C_{1}\left(\left\|f_{t^{l}}(\cdot, \tau)\right\|_{L_{2}(\Omega)}^{2}+\int_{h}^{\tau}\left\|f_{t^{l+1}}(\cdot, t)\right\|_{L_{2}(\Omega)}^{2} d t\right) \\
+\epsilon_{1}\left(\left\|v_{t^{l}}^{N}(\cdot, \tau)\right\|_{H^{m}(\Omega)}^{2}+\int_{h}^{\tau}\left\|v_{t^{l}}^{N}(\cdot, t)\right\|_{H^{m}(\Omega)}^{2} d t\right),
\end{gathered}
$$

where $C_{1}=\max \left\{\frac{\mu m^{\star} M}{\epsilon_{1}}, \frac{1}{\epsilon_{1}}\right\}, M=\max _{s=0, \ldots, l-1}\left(\begin{array}{l}l \\ s\end{array}\right)$.

Denote $\epsilon=\left(\left(2^{l+1}-2-l\right) \mu m^{\star}+1\right) \epsilon_{1}>0$ for $l>0$, then for all $0<\epsilon<\mu_{0}$, using above estimations and (3.5), we obtain

$$
\begin{aligned}
& \left\|v_{t^{l}}^{N}(\cdot, \tau)\right\|_{H^{m}(\Omega)}^{2} \leq \frac{(2 l+1) \mu m^{\star}+\epsilon}{\mu_{0}-\epsilon} \\
& :=\alpha_{l} \int_{h}^{\tau}\left\|v_{t^{l}}^{N}(\cdot, t)\right\|_{H^{m}(\Omega)}^{2} d t+C \cdot \sum_{k=0}^{l-1}\left\|v_{t^{k}}^{N}(\cdot, \tau)\right\|_{H^{m}(\Omega)}^{2} \\
& +C \cdot \sum_{k=0}^{l-1} \int_{h}^{\tau}\left\|v_{t^{k}}^{N}(\cdot, t)\right\|_{H^{m}(\Omega)}^{2} d t \\
& +C \cdot\left\|f_{t^{l}}(\cdot, \tau)\right\|_{L_{2}(\Omega)}^{2}+C \cdot \int_{h}^{\tau}\left\|f_{t^{l+1}}(\cdot, t)\right\|_{L_{2}(\Omega)}^{2} d t,
\end{aligned}
$$

where $C$ is a positive constant.

On the other hand, in [6] one already has the following estimate:

$$
\begin{aligned}
\left\|v^{N}(\cdot, \tau)\right\|_{H^{m}(\Omega)}^{2} \leq & 2 \alpha \int_{h}^{\tau} e^{2 \alpha(\tau-t)}\left(\|f(\cdot, t)\|_{L_{2}(\Omega)}^{2}+\int_{h}^{\tau}\left\|f_{s}(\cdot, s)\right\|_{L_{2}(\Omega)}^{2} d s\right) d t \\
& +C\left(\|f(\cdot, \tau)\|_{L_{2}(\Omega)}^{2}+\int_{h}^{\tau}\left\|f_{t}(\cdot, t)\right\|_{L_{2}(\Omega)}^{2} d t\right) .
\end{aligned}
$$

Multiplying both sides of (3.7) by $e^{-2 \gamma \tau}$, then integrating with respect to $\tau$ from $h$ to $\infty$ we obtain

$$
\left\|v^{N}\right\|_{H^{m, 0}\left(-\gamma, \Omega_{h}^{\infty}\right)}^{2} \leq C\left(\|f\|_{L_{2}\left(-\gamma, \Omega_{h}^{\infty}\right)}^{2}+\left\|f_{t}\right\|_{L_{2}\left(-\gamma, \Omega_{h}^{\infty}\right)}^{2}\right)
$$

where the constant $C$ does not depend on $h$. Now using (3.8) and (3.6) for $l=1$ yields

$$
\begin{aligned}
\left\|v_{t}^{N}\right\|_{H^{m, 0}\left(-\gamma, \Omega_{h}^{\infty}\right)}^{2} & \\
\leq & C\left(\left\|f_{t}\right\|_{L_{2}\left(-\gamma, \Omega_{h}^{\infty}\right)}^{2}+\left\|f_{t^{2}}\right\|_{L_{2}\left(-\gamma, \Omega_{h}^{\infty}\right)}^{2}+\left\|v^{N}\right\|_{H^{m, 0}\left(-\gamma, \Omega_{h}\right)}^{\infty}\right) \\
& +C \cdot \int_{h}^{\infty} e^{-2 \gamma \tau} \int_{h}^{\tau} e^{\alpha_{1}(\tau-t)}\left(\left\|v^{N}(\cdot, t)\right\|_{H^{m}(\Omega)}^{2}+\left\|f_{t}(\cdot, t)\right\|_{L_{2}(\Omega)}^{2}\right) d t d \tau \\
& +C \cdot \int_{h}^{\infty} e^{-2 \gamma \tau} \int_{h}^{\tau} e^{\alpha_{1}(\tau-t)} \int_{h}^{t}\left(\left\|v^{N}(\cdot, s)\right\|_{H^{m}(\Omega)}^{2}+\left\|f_{t^{2}}(\cdot, s)\right\|_{L_{2}(\Omega)}^{2}\right) d s d t d \tau .
\end{aligned}
$$

Remember that

$$
\inf _{\mu_{0}>\epsilon>0} 2 \alpha_{1}=\inf _{\mu_{0}>\epsilon>0} \frac{3 \mu m^{\star}+\epsilon}{\mu_{0}-\epsilon}=6 \gamma_{0}<2 \gamma .
$$


So we can choose $\epsilon$ small enough such that $\alpha_{1}<\gamma$. By (3.9) and using the Fubini theorem we obtain

$$
\left\|v_{t}^{N}\right\|_{H^{m, 0}\left(-\gamma, \Omega_{h}^{\infty}\right)}^{2} \leq C\left(\sum_{k=0}^{2}\left\|f_{t^{k}}\right\|_{L_{2}\left(-\gamma, \Omega_{h}^{\infty}\right)}^{2}+\left\|v^{N}\right\|_{H^{m, 0}\left(-\gamma, \Omega_{h}^{\infty}\right)}^{2}\right),
$$

which implies

$$
\left\|v_{t}^{N}\right\|_{H^{m, 0}\left(-\gamma, \Omega_{h}^{\infty}\right)}^{2} \leq C \sum_{k=0}^{2}\left\|f_{t^{k}}\right\|_{L_{2}\left(-\gamma, \Omega_{h}^{\infty}\right)}^{2} .
$$

Repeating the above arguments, from (3.6) one can prove for any $l$, if $f_{t^{k}} \in L_{2}\left(-\gamma, \Omega_{h}^{\infty}\right)$, $k=\overline{0, l+1}$, that $v^{N}$ has derivative with respect to $t$ up to the $l$ th and for any $k=\overline{0, l}$

$$
\left\|v_{t^{k}}^{N}\right\|_{H^{m, 0}\left(-\gamma, \Omega_{h}^{\infty}\right)}^{2} \leq C \sum_{m_{1}=0}^{k+1}\left\|f_{t^{m_{1}}}\right\|_{L_{2}\left(-\gamma, \Omega_{h}^{\infty}\right)}^{2} .
$$

The inequality (3.11) implies that $\left\{v_{t^{k}}^{N}\right\}$ is uniformly bounded in $H^{m, 0}\left(-\gamma, \Omega_{h}^{\infty}\right)$. By a standard weakly convergence argument, we can conclude that the sequence $\left\{v_{t^{k}}^{N}\right\}_{N=1}^{\infty}$ possesses a subsequence convergent to a vector function $v_{t^{k}}$ in $H^{m, 0}\left(-\gamma, \Omega_{h}^{\infty}\right)$. Moreover, it follows from (3.11) that (3.1) holds.

\section{Further results on the regularity of solution of problem with initial condition}

Assume that $\omega$ is a local coordinate system on $S^{n-1}$. Moreover, assume that the principal part of the operator $L(x, t, D)$ at origin 0 can be written in the form

$$
L_{0}(0, t, D)=r^{-2 m} Q\left(\omega, t, r D_{r}, D_{\omega}\right), \quad D_{r}=\frac{i \partial}{\partial r}
$$

where $Q$ is a linear operator with smooth coefficients. Consider the following spectral problem:

$$
\begin{aligned}
& Q\left(\omega, t, \lambda, D_{\omega}\right) v(\omega)=0, \quad \omega \in G ; \\
& D_{\omega}^{j} v(\omega)=0, \quad \omega \in \partial G, j=0, \ldots, m-1 .
\end{aligned}
$$

Proceeding similarly to Lemma 2.1 in [1], the following lemma holds.

Lemma 4.1 Let $f, f_{t}, f_{t t} \in L_{2}\left(-\gamma, K_{h}^{\infty}\right), \gamma>(4 m+1) \gamma_{0}$ and $f(x, h)=f_{t}(x, h)=0$. If $v(x, t)$ is a generalized solution of problem (2.5)-(2.7) in the space $\stackrel{\circ}{H}^{m, 0}\left(-\gamma, K_{h}^{\infty}\right)$ such that $u \equiv 0$ whenever $|x|>R, R=$ const., then $v \in H_{m}^{2 m, 1}\left(-\gamma, K_{h}^{\infty}\right)$ and the following estimate holds:

$$
\|v\|_{H_{m}^{2 m, 1}\left(-\gamma, K_{h}^{\infty}\right)}^{2} \leq C\left[\|f\|_{L_{2}\left(-\gamma, K_{h}^{\infty}\right)}^{2}+\left\|f_{t}\right\|_{L_{2}\left(-\gamma, K_{h}^{\infty}\right)}^{2}+\left\|f_{t t}\right\|_{L_{2}\left(-\gamma, K_{h}^{\infty}\right)}^{2}\right]
$$

where the constant $C$ is independent of $v, f, h$.

Lemma 4.2 Let $v(x, t)$ be a generalized solution of problem (2.5)-(2.7), $\gamma>(4 m+1) \gamma_{0}$ and let $f_{t^{k}} \in L_{2}\left(-\gamma, K_{h}^{\infty}\right)$ for $k \leq 2 m+1, f_{t^{k}}(x, h)=0$ for $k \leq 2 m$. In addition suppose that the 
strip

$$
m-\frac{n}{2} \leq \operatorname{Im} \lambda \leq 2 m-\frac{n}{2}
$$

does not contain points of the spectrum of problem (4.1)-(4.2) for every $t \in[h, \infty)$. Then $v \in H_{0}^{2 m}\left(-\gamma, K_{h}^{\infty}\right)$ and the following estimate holds:

$$
\|v\|_{H_{0}^{2 m}\left(-\gamma, K_{h}^{\infty}\right)}^{2} \leq C \sum_{k=0}^{2 m+1}\left\|f_{t^{k}}\right\|_{L_{2}\left(-\gamma, K_{h}^{\infty}\right)}^{2}
$$

where the constant $C$ is independent of $v, f, h$.

Proof First, we prove that

$$
\left\|v_{t^{s}}\right\|_{H_{0}^{2 m, 0}\left(-\gamma, K_{h}^{\infty}\right)}^{2} \leq C \sum_{k=0}^{2 m+1}\left\|f_{t^{k}}\right\|_{L_{2}\left(-\gamma, K_{h}^{\infty}\right)}^{2}
$$

where $C$ does not depend on $h, s \leq 2 m$.

Doing the same in [1, Proposition 2.1] we have

$$
\|v\|_{H_{0}^{2 m}(K)}^{2} \leq C\left[\|f\|_{L_{2}(K)}^{2}+\left\|v_{t}\right\|_{L_{2}(K)}^{2}+\|v\|_{H_{m}^{2 m}(K)}^{2}\right]
$$

for a.e. $t \in[h, \infty)$, where $C=$ constant.

Multiplying both sides of this inequality by $e^{-2 \gamma t}$, then integrating with respect to $t$ from $h$ to $\infty$, we get

$$
\|v\|_{H_{0}^{2 m, 0}\left(-\gamma, K_{h}^{\infty}\right)}^{2} \leq C\left[\|f\|_{L_{2}\left(-\gamma, K_{h}^{\infty}\right)}^{2}+\left\|v_{t}\right\|_{L_{2}\left(-\gamma, K_{h}^{\infty}\right)}^{2}+\|v\|_{H_{0}^{2 m, 0}\left(-\gamma, K_{h}^{\infty}\right)}^{2}\right] .
$$

By (3.11)

$$
\left\|v_{t}\right\|_{L_{2}\left(-\gamma, K_{h}^{\infty}\right)}^{2} \leq C \sum_{k=0}^{2}\left\|f_{t^{k}}\right\|_{L_{2}\left(-\gamma, K_{h}^{\infty}\right)}^{2}
$$

and by Lemma 4.1

$$
\|v\|_{H_{0}^{2 m, 0}\left(-\gamma, K_{h}^{\infty)}\right.}^{2} \leq\|v\|_{H_{m}^{2 m, 1}\left(-\gamma, K_{h}^{\infty}\right)}^{2} \leq C \sum_{k=0}^{2}\left\|f_{t^{k}}\right\|_{L_{2}\left(-\gamma, K_{h}^{\infty}\right)}^{2},
$$

from which it follows that

$$
\|v\|_{H_{0}^{2 m, 0}\left(-\gamma, K_{h}^{\infty}\right)}^{2} \leq C \sum_{k=0}^{2}\left\|f_{t^{k}}\right\|_{L_{2}\left(-\gamma, K_{h}^{\infty}\right)}^{2} .
$$

That means (4.5) is proved for $s=0$. 
Now assume that (4.5) is true for $s-1$. By differentiating the systems (2.5) $s$ times with respect to $t$ and by putting $w=v_{t}$, we obtain

$$
(-1)^{m-1} L w=-i\left(w_{t}+f_{t^{s}}\right)+(-1)^{m} \sum_{k=1}^{s}\left(\begin{array}{l}
s \\
k
\end{array}\right) L_{t^{k}} v_{t^{s-k}}
$$

where $L_{t^{k}}=\sum_{|p|,|q|=0}^{m} D^{p}\left(\frac{\partial^{k} a_{p q}}{\partial t^{k}} D^{q}\right)$.

From the inductive hypothesis and repeating the arguments of the proof in the case $s=0$, the inequality (4.5) holds for $s$.

Since

$$
\|v\|_{H_{0}^{2 m}\left(-\gamma, K_{h}^{\infty}\right)}^{2} \leq C\left(\sum_{s=0}^{2 m-1}\left\|v_{t^{s}}\right\|_{H_{0}^{2 m, 0}\left(-\gamma, K_{h}^{\infty}\right)}^{2}+\| v_{t^{2 m} \|_{H_{0}^{0,0}\left(-\gamma, K_{h}^{\infty}\right)}^{2}}\right)
$$

from (4.5) and Theorem 4.1, (4.4) is true. The lemma is proved.

Theorem 4.1 Let $l$ be a nonnegative integer. Assume that $v(x, t)$ is a weak solution in the space $H^{m, 0}\left(-\gamma, \Omega_{h}^{\infty}\right)$ with $\gamma>(2(2 m+l)+1) \gamma_{0}$ of problem $(2.5)-(2.7)$ and $f_{t^{k}} \in H_{0}^{l, 0}\left(-\gamma, \Omega_{h}^{\infty}\right)$, for $k \leq 2 m+l+1, f_{t^{k}}(x, h)=0$ for $k \leq 2 m+l$. In addition, suppose that in the strip

$$
m-\frac{n}{2} \leq \operatorname{Im} \lambda \leq 2 m+l-\frac{n}{2}
$$

there is no point from the spectrum of (4.1)-(4.2). Then we have $v \in H_{0}^{2 m+l}\left(-\gamma, \Omega_{h}^{\infty}\right)$ and the following estimate holds:

$$
\|v\|_{H_{0}^{2 m+l}\left(-\gamma, \Omega_{h}^{\infty}\right)}^{2} \leq C \sum_{k=0}^{2 m+l+1}\left\|f_{t^{k}}\right\|_{H_{0}^{l, 0}\left(-\gamma, \Omega_{h}^{\infty}\right)}^{2}
$$

Proof 1 . Firstly, we study the case $u \equiv 0$ outside $U_{0}$.

We use the induction by $l$. For $l=0$, this theorem is proved by Lemma 4.2 with noting that $H_{0}^{0,0}\left(-\gamma, K_{h}^{\infty}\right)=L_{2}\left(-\gamma, K_{h}^{\infty}\right)$. Assume that the theorem's assertion holds up to $l-1$, we need to prove that this holds up to $l$.

Firstly we will prove the following inequality:

$$
\left\|v_{t}\right\|_{H_{0}^{2 m+l-j}}^{2}\left(-\gamma, K_{h}^{\infty}\right) \leq C \sum_{k=0}^{2 m+l+1}\left\|f_{t^{k}}\right\|_{H_{0}^{l, 0}\left(-\gamma, K_{h}^{\infty}\right)}^{2}
$$

for all $j=l, l-1, \ldots, 0$, where the constant $C$ is independent of $h$.

Since $f_{t^{k}} \in H_{0}^{l}\left(-\gamma, K_{h}^{\infty}\right), f_{t^{k}} \in L_{2}\left(-\gamma, K_{h}^{\infty}\right)$. So using similar arguments in the proof of Theorem 4.1 we get $v_{t} \in H^{2 m, 0}\left(-\gamma, K_{h}^{\infty}\right)$. By Lemma 4.2, one obtains $v_{t^{l}} \in H_{0}^{2 m}\left(-\gamma, K_{h}^{\infty}\right)$. This means that (4.7)holds for $j=l$.

Assume that (4.7) holds for $j=l, l-1, \ldots, s+1$.

By the assumption of the induction of $l, v \in H_{0}^{2 m+l-1}\left(-\gamma, K_{h}^{\infty}\right)$, put $w=v_{t^{s}}$ then $w \in$ $H_{0}^{2 m+l-s-1}\left(-\gamma, K_{h}^{\infty}\right)$. Differentiating (2.5) $s$-times with respect to $t$, we have

$$
L w=F=-i\left(w_{t}+f_{t^{s}}\right)+\sum_{p=1}^{s}\left(\begin{array}{l}
s \\
p
\end{array}\right) L_{t} p v_{t^{s-p}}
$$


where $L_{t^{k}}=\sum_{|p|,|q|=0}^{m}(-1)^{|p|} D^{p}\left(\frac{\partial^{k} a_{p q}}{\partial t^{k}} D^{q}\right)$. Following the assumption of the induction of $j$ and the hypothesis of the function $f$ one has $w_{t} \in H_{0}^{2 m+l-s-1}\left(-\gamma, K_{h}^{\infty}\right), f_{t^{s}} \in H_{0}^{l-s}\left(-\gamma, K_{h}^{\infty}\right)$. It follows that $F \in H_{0}^{l-s}\left(-\gamma, K_{h}^{\infty}\right) \subset H_{-1}^{l-s-1}\left(-\gamma, K_{h}^{\infty}\right)$. Because the strip $2 m+l-s-1-\frac{n}{2} \leq$ $\operatorname{Im} \lambda \leq 2 m+l-s-\frac{n}{2}$ does not contain spectral points of problem (4.1)-(4.2), then using results in [8], one gets $w \in H_{-1}^{2 m+l-s-1}(K)$, a.e. $t \in(h, \infty)$. So $w \in H_{-1}^{2 m+l-s-1,0}\left(-\gamma, K_{h}^{\infty}\right)$. Note that $F \in H_{0}^{l-s}\left(-\gamma, K_{h}^{\infty}\right)$, then by using similar method to the one used in Lemma 2.2 in [1] we have $w \in H_{-1}^{2 m+l-s-1,0}\left(-\gamma, K_{h}^{\infty}\right)$ and the following estimate holds:

$$
\|w\|_{H_{-1}^{2 m+l-s-1,0}\left(-\gamma, K_{h}^{\infty}\right)}^{2} \leq C\left[\|F\|_{H_{0}^{l-s, 0}}^{2}+\|w\|_{H_{-1}^{l-s+1,0}\left(-\gamma, K_{h}^{\infty}\right)}^{2}\right]
$$

where $C$ is a constant independent of $h$.

So using the inductive assumption and (4.9), we obtain

$$
\left\|v_{t^{j}}\right\|_{H_{0}^{2 m+l-s}}^{2}\left(-\gamma, K_{h}^{\infty}\right) \leq C \sum_{k=0}^{2 m+l+1}\left\|f_{t^{k}}\right\|_{H_{0}^{l, 0}\left(-\gamma, K_{h}^{\infty}\right)}^{2} .
$$

It means that (4.7) is proved and our theorem is completed by fixing $j=0$ in (4.7).

2. The general case: Consider a function $\varphi_{0} \in C_{0}^{\infty}\left(U_{0}\right)$ such that $\varphi_{0} \equiv 1$ in some neighborhood of 0 . Definite $v_{0}=\varphi_{0} \nu$, which satisfies the system

$$
(-1)^{m-1} i L(x, t, D) v_{0}-\left(v_{0}\right)_{t}=\varphi_{0} f+L^{\prime}(x, t, D) v,
$$

where $L^{\prime}(x, t, D)$ is a linear operator having order less than $2 m$ and the coefficients of this operator is equal to 0 outside $U_{0}$. So the first case of this theorem implies that

$$
\left\|v_{0}\right\|_{H_{0}^{2 m+l}\left(-\gamma, \Omega_{h}^{\infty}\right)}^{2} \leq C \sum_{k=0}^{2 m+l+1}\left\|f_{t^{k}}\right\|_{H_{0}^{l, 0}\left(-\gamma, \Omega_{h}^{\infty}\right)}^{2} .
$$

Write $v=v_{0}+v_{1}$ where $v_{1}=\left(1-\varphi_{0}\right) v$. The function $v_{1}$ is equal to 0 in $U_{0}$, so we can apply the famous results on the smoothness of solutions of Schrödinger systems in a smooth domain to this function and obtain

$$
\left\|v_{1}\right\|_{H_{0}^{2 m+l}\left(-\gamma, \Omega_{h}^{\infty}\right)}^{2} \leq C \sum_{k=0}^{2 m+l+1}\left\|f_{t^{k}}\right\|_{H_{0}^{l, 0}\left(-\gamma, \Omega_{h}^{\infty}\right)}^{2} .
$$

From inequalities (4.11) and (4.12) it follows that

$$
\|v\|_{H_{0}^{2 m+l}\left(-\gamma, \Omega_{h}^{\infty}\right)}^{2} \leq C \sum_{k=0}^{2 m+l+1}\left\|f_{t^{k}}\right\|_{H_{0}^{l, 0}\left(-\gamma, \Omega_{h}^{\infty}\right)}^{2} .
$$

The theorem is proved.

\section{The regularity of solution of problem (2.2)-(2.3)}

The generalized solution of problem (2.2)-(2.3) can be approximated by a sequence of solutions of problems with initial condition (2.5)-(2.7). 
It is well known that there is a smooth function $\chi(t)$ which is equal to 1 on $[1, \infty)$, is equal to 0 on $(-\infty, 0]$, and assumes value in $[0,1]$ on $[0 ; 1]$ (see [9, Th. 5.5] for more details). Moreover, we can suppose that all derivatives of $\chi(t)$ are bounded.

Let $h \in(-\infty, 0]$ be an integer. We set $f^{h}(x, t)=\chi(t-h) f(x, t)$ and we consider a generalized solution $u^{h}$ and $u^{j}$ of problems (2.5)-(2.7) in cylinders $\Omega_{h}^{\infty}$ and $\Omega_{j}^{\infty}$ with $f(x, t)$ replaced by $f^{h}(x, t)$ and $f^{j}(x, t)$, respectively. If $h>j, u^{h}$ can be defined in $\stackrel{\circ}{H}^{m, 0}\left(-\gamma, \Omega_{j}^{\infty}\right)$ with $u^{h}(x, t)=0, \forall j \leq t \leq h$, then we put $u^{j h}=u^{j}(x, t)-u^{h}(x, t)$, and $u^{j h}$ is the generalized solution of problem (2.5)-(2.7) in which $f(x, t)$ is replaced by $f^{j h}=f^{j}(x, t)-f^{h}(x, t)$. According to (4.6),

$$
\left\|u_{t^{k}}^{j h}\right\|_{H_{0}^{2 m+l}\left(-\gamma, \Omega_{j}^{\infty}\right)}^{2}=\left\|u_{t^{k}}^{j h}\right\|_{H_{0}^{2 m+l}\left(-\gamma, \Omega_{\mathbb{R}}\right)}^{2} \leq C \sum_{m_{1}=0}^{2 m+l+1}\left\|f_{t^{m_{1}}}^{j h}\right\|_{H_{0}^{l, 0}\left(-\gamma, \Omega_{j}^{\infty}\right)}^{2} .
$$

Because

$$
\begin{aligned}
\left\|f^{j h}\right\|_{H^{l, 0}\left(-\gamma, \Omega_{j}^{\infty}\right)}^{2} & =\int_{j}^{h+1} e^{-2 \gamma t}\left\|f^{h}-f^{j}\right\|_{H_{0}^{l}(\Omega)}^{2} d t \\
& =\int_{j}^{h+1} e^{-2 \gamma t}|\chi(t-h)-\chi(t-j)|\|f\|_{H_{0}^{l}(\Omega)}^{2} d t \\
& \leq 2 \int_{j}^{h+1} e^{-2 \gamma t}\|f\|_{H_{0}^{l}(\Omega)}^{2} d t .
\end{aligned}
$$

Since $f \in H_{0}^{l, 0}\left(-\gamma, \Omega_{\mathbb{R}}\right), \quad \lim \int_{j}^{h+1} e^{-2 \gamma t}\|f\|_{H_{0}^{l}(\Omega)}^{2} d t=0 \quad$ when $h, j \rightarrow-\infty$. So $\lim \left\|f^{j h}\right\|_{H_{0}^{l, 0}\left(-\gamma, \Omega_{j}^{\infty}\right)}^{2}=0$ when $h, j \rightarrow-\infty$. Repeating this argument, we discover $\lim \left\|f_{t^{m_{1}}}^{j h}\right\|_{H_{0}^{l, 0}\left(-\gamma, \Omega_{j}^{\infty}\right)}^{2}=0$ when $h, j \rightarrow-\infty$, for all $m_{1}=0, \ldots, k+1$. It follows that $\left\{u_{t^{k}}^{h}\right\}_{h=0}^{-\infty}$ is a Cauchy sequence and $u_{t^{k}}^{h}$ is convergent to $u_{t^{k}}$ in $H_{0}^{2 m+l}\left(-\gamma, \Omega_{\mathbb{R}}\right)$. Moreover, $H_{0}^{2 m+l}\left(-\gamma, \Omega_{\mathbb{R}}\right)$ is continuously embedded in $H^{m, 0}\left(-\gamma, \Omega_{\mathbb{R}}\right)$, since

$$
\|u\|_{H_{0}^{2 m+l}\left(-\gamma, \Omega_{\mathbb{R}}\right)}^{2} \leq \sum_{|\alpha|=0}^{2 m+l} \int_{\Omega_{\mathbb{R}}} e^{-2 \gamma t} r^{2(|\alpha|-2 m-l)}\left|D^{\alpha} u\right|^{2} \leq C\|u\|_{H^{m, 0}\left(-\gamma, \Omega_{\mathbb{R}}\right)}^{2}
$$

So it is very easy to verify that $u \in H^{m, 0}\left(-\gamma, \Omega_{\mathbb{R}}\right)$ is a generalized solution of problem (2.2)(2.3); see [7]. We obtain the following main results.

Theorem 5.1 Assume that $l \in \mathbb{N}$ and there exists $\mu>0$ such that

(i) $\sup \left\{\left|\frac{\partial a_{p q}}{\partial t}\right|:(x, t) \in \Omega_{\mathbb{R}}, 0 \leq|p|,|q| \leq m,\right\}=\mu<\infty,\left|\frac{\partial^{k} a_{p q}}{\partial t^{k}}\right| \leq \mu_{2}, \mu_{2}>0$, for $2 \leq k \leq l+1$

(ii) $\left|\frac{\partial a_{p q}}{\partial t}\right| \leq \mu_{1} \cdot e^{2 \gamma t}$, for all $(x, t) \in \Omega_{\mathbb{R}}, 0 \leq|p|,|q| \leq m$;

(iii) $f_{t^{k}} \in L_{2}\left(-\gamma, \Omega_{\mathbb{R}}\right)$, for all $x \in \Omega, 0 \leq k \leq l+1$.

Then for all $\gamma>(2 l+1) \gamma_{0}$, the solution $u \in \stackrel{\circ}{H}^{m, 0}\left(-\gamma, \Omega_{\mathbb{R}}\right)$ of problem (2.2)-(2.3) has derivatives with respect to $t$ up to order $l$ satisfying $u_{t^{k}} \in H^{m, 0}\left(-\gamma, \Omega_{\mathbb{R}}\right), \forall k=\overline{0, l}$, such that

$$
\left\|u_{t^{k}}\right\|_{H^{m, 0}\left(-\gamma, \Omega_{\mathbb{R}}\right)}^{2} \leq C \sum_{m_{1}=0}^{k+1}\left\|f_{t^{m_{1}}}\right\|_{L_{2}\left(-\gamma, \Omega_{\mathbb{R}}\right)}^{2}
$$

where the constant $C$ does not depend on $u, f$. 
Theorem 5.2 Let $l$ be a nonnegative integer. Assume that $u(x, t)$ is a weak solution in the space $H^{m, 0}\left(-\gamma, \Omega_{\mathbb{R}}\right)$ with $\gamma>(2(2 m+l)+1) \gamma_{0}$ of the problem (2.2)-(2.3) and $f_{t^{k}} \in$ $H_{0}^{l, 0}\left(-\gamma, \Omega_{\mathbb{R}}\right)$, for $k \leq 2 m+l+1$. In addition, suppose that in the strip

$$
m-\frac{n}{2} \leq \operatorname{Im} \lambda \leq 2 m+l-\frac{n}{2}
$$

there is no point from the spectrum of (4.1)-(4.3). Then we have $u \in H_{0}^{2 m+l}\left(-\gamma, \Omega_{\mathbb{R}}\right)$ and the following estimate holds:

$$
\|u\|_{H_{0}^{2 m+l}\left(-\gamma, \Omega_{\mathbb{R}}\right)}^{2} \leq C \sum_{k=0}^{2 m+l+1}\left\|f_{t^{k}}\right\|_{H_{0}^{l, 0}\left(-\gamma, \Omega_{\mathbb{R}}\right)}^{2}
$$

\section{Competing interests}

The authors declare that they have no competing interests.

\section{Authors' contributions}

All authors read and approved the final manuscript.

\section{Author details}

${ }^{1}$ Department of Mathematics, Hanoi National University of Education, Hanoi, Vietnam. ${ }^{2}$ National Institute of Education Management, Hanoi, Vietnam.

\section{Acknowledgements}

We would like to thank the reviewers for a careful reading and valuable comments on the original manuscript.

Received: 25 March 2014 Accepted: 4 July 2014 Published online: 24 September 2014

\section{References}

1. Hung, NM, Anh, CT: On the smoothness of solutions of the first initial boundary value problem for Schrödinger systems in domain with conical points. Vietnam J. Math. 33(2), 135-147 (2005)

2. Hung, NM, Son, NTK: On the regularity of solution of the second initial boundary value problem for Schrodinger systems in domains with conical points. Taiwan. J. Math. 13(6), 1885-1907 (2009)

3. Bokalo, NM: Dynamical problems without initial conditions for elliptic-parabolic equations in spatial unbounded domains. Electron. J. Differ. Equ. 2010, 178 (2010)

4. Bokalo, NM: Problem without initial conditions for some classes of nonlinear parabolic equations. J. Sov. Math. 51, 2291-2322 (1990)

5. Bokalo, M, Dmytryshyn, Y: Problems without initial conditions for degenerate implicit evolution equations. Electron. J. Differ. Equ. 2008, 04 (2008)

6. Moiseev, El, Vafodorova, GO: Problems without initial conditions for some differential equations. Differ. Equ. 38(8), $1162-1165$ (2002)

7. Hung, NM, Lien, NT: On the solvability of the boundary value problem without initial condition for Schrödinger systems in infinite cylinders. Bound. Value Probl. 2013, 156 (2013)

8. Maz'ya, VG, Plamenevskii, BA: On the coefficients in the asymptotic of solutions of the elliptic boundary problem in domains with conical points. Transl. Am. Math. Soc. 123, 57-88 (1984). Translated from: Math. Nachr. 76, $29-60$ (1977)

9. Adams, RA: Sobolev Spaces. Academic Press, San Diego (1975) 